\title{
Feasibility Study for Neutron Beam Investigation of the Turin Shroud
}

\author{
Massimo Rogante, László Rosta
}

\begin{abstract}
In this work, the possible investigations and analyses of the Turin Shroud by Neutron Beam Techniques (NBT) are discussed. The main aim of this study is to assess - in a reliable non-destructive way and in complementarity to methods already employed or under consideration for future examination campaigns - and to determine some key parameters useful to enhance current knowledge and databases on the textile properties of the Shroud. Information to be obtained could reveal new interesting and original features with potential scientific and historical effects as well as contributing to solving problems of conservation and preservation. Preliminary results of NBT analyses carried out on new and aged linen fibres illustrate some information that could be gained when using NBT on the Turin Shroud.
\end{abstract}

Index Terms - Turin Shroud, Neutron beam techniques, SANS, Neutron diffraction.

\section{INTRODUCTION}

The Turin Shroud (TS) is a $4.4 \times 1.1 \mathrm{~m}$ linen cloth supposed to be the burial Holy Shroud of Jesus Christ. The state of the art concerning the investigation of the Shroud has allowed the scientists involved in this activity to confirm that the Shroud's body image is definitely not a painting, as demonstrated by already well-known chemical, physical and computer investigations. Several examinations have been carried out, starting from the year 1978, in particular within the framework of the Shroud of Turin Research Project (STURP): a variety of independent experienced investigators conducted a round-the-clock examination for 120 hours, applying different techniques to study the fabric as well as the frontal and the body image on its surface [1]. Forensic, image, chemical and spectroscopic investigations were performed, as well as detailed analyses of the possible resolution of the various conservation and preservation issues. The status of the TS, nevertheless, is debated, since a questionable radiocarbon examination carried out with ${ }^{14}$ Carbon on a specific sample of the cloth dated it in the 14 th century, from $1325 \pm 65$ years. The procedures adopted for the ${ }^{14} \mathrm{C}$ examination have been controversially discussed (e.g., chemical and other issues could have corrupted the date) and the plan of a new complementary examination campaign is currently being considered. In particular, information on the nanoscale of the TS is not complete, including the nanoscaled features able to differentiate the ancient and modern linen fabrics: these information are still lacking.

Linen is made from the fibres of the flax plant (Linum usitatissimum) and its textiles seem to be some of the oldest in the world: their past goes back several thousands of years. These fibres are composed of pure cellulose fibrils associated with complementary substances (hemicellulose, lignin, pectin) that form inter-fibre lamellae and cementing textiles, which go with the cellulose up to the finished product. Their number varies depending on the conditions of production and processing. It is also possible that their distribution along the fibres axis is inhomogeneous [2]. Important properties of these fibres are: the crystallites dimensions and crystallinity, the homogeneity and shape over the cross section, the orientation degree, the pore dimensions, size and volume, the internal surface and the molecular flexibility inside the fibre at dissimilar situations. Scattering methods (in particular, X-ray scattering) have been widely employed in the past, to study the structure of cellulose fibres, determining orientation and sizes of crystallites and voids [3]. Nevertheless, X-rays are not able to characterize the sub-structures.

Neutron Beam Techniques (NBT) have come to the fore recently as an effective tool for characterising materials and components across a range of disciplines, including the Cultural Heritage sector. These methods reveal significant properties and allow the assessment of responses to external influences over the sample's entire volume [4, 5]. What differentiates NBT from other investigations is the capability to non-destructively capture information about the material's micro- and nano-physical structures down to $\sim 1$ $\AA \quad(0.1 \mathrm{~nm})$ [4]. The present feasibility study, after presenting a state-of-the-art related to the analysis of the TS, concerns a potential investigation of the TS by NBT - in particular, Small Angle Neutron Scattering (SANS) and Neutron Diffraction (ND) - in order to supply the researchers, complementarily with the methodologies adopted in the past or employable in eventual upcoming examination campaigns, with fundamental parameters helpful and suitable to be inserted in the complex information mosaic to comprehend the mystery of the Sindonic Image. SANS technique has already been tested on new and aged linen fabrics dated from Old Kingdom to Ptolemaic ages (2200-300 B.C.), confirming the possibility to get interesting results when using NBT on the Turin Shroud.

Massimo Rogante, Rogante Engineering Office, Contrada San Michele, 61, 62012 Civitanova Marche, Italy.

László Rosta, Wigner Research Centre for Physics, Institute for Solid State Physics and Optics, Pf. 49, 1525 Budapest, Hungary. 


\section{MAJOR SCIENTIFIC INVESTIGATIONS ALREADY CARRIED OUT AND RESULTS}

\section{A. Photographic studies}

Photographic studies in several regions of the electromagnetic spectrum were carried out [6], with further several form of analysis by different types of computer algorithms. Colour microphotographs were made and, as a result, it was found that the straw yellow colour of the body image fibres does not match the colour of any of the known forms of ferric iron oxides. Albedo (simple reflection) images, phosphorescent emission images, chemical contact images, thermal imaging, diffusion images, electrostatic imaging, dry powder contact images and scorching contact with an engraving were made. Polarized image overlay techniques were adopted also, to facilitate and better quantify the comparisons of images in iconographic studies. Using appropriate light sources and filters, a series of UV fluorescent photographic images of the Shroud were made and compared to colour reflectance photographic images taken of the same areas [7].

- Reflection and transmission photographs. Appropriate light sources and filters were adopted, to obtain a series of ultraviolet fluorescent photographic images, then compared to colour reflectance photographic images taken from the same areas [8].

- Infrared thermography, to examine paintings in order to detect evidence of any underlining paint structures or of a painter outlining his composition. Neither of these kinds of evidence was revealed, in agreement with the conclusion that the body images are not paintings [9].

- Ultraviolet emission. Ultraviolet-visible reflectance and fluorescence. The visible reflectance spectra and the red-green-blue characteristics of the body images were a closer match to the lightly scorched areas (and oxidized cellulose area) than to controls of linen with an iron oxide coating. The near UV spectra showed a broad band assignable to those observed for conjugated carbonyl absorption $[10,11]$.

- X-ray Absorptiometry (Radiography) by medical type diagnostic instrument. High quality films were produced, with weave structure, cloth density variations, water stain margins and repair panel stitching all clearly visible. Adequateness was confirmed to resolve the threads in the linen cloth. The diameter of individual threads was estimated of the order of $0.15 \mathrm{~mm}$, compared to a range of 0.1-0.45 mm for the backing cloth. High-density inclusions were scattered throughout the Shroud [12].

- Spectrophotometry, Photoelectric Spectral Reflectometry and Fluorimetry. Normalized "absolute" reflectance measurements provided the true colours of the Shroud areas. An analysis of various body image areas (e.g., nose, cheek, calf and neck) was carried out and reflectance measurements were made on selected blood spots. In the photoelectric measurements, the fluorescence of the linen dominates and obscures signals appear that might be characteristic of the other types of stain [13].

\section{B. Computer image analysis}

Computer image analysis - also using Fourier transform -, to obtain particular domain of interest. An analysis of graphic signs and a three dimensional relief were carried out, with application of appropriate filters to the wounded image which was subdivided into areas characterized by an intrinsic distribution of luminance; an analysis of imprints of coins on the face was also performed [14].

\section{Chemical investigations}

Chemical investigations on material removed by various sampling techniques (mainly by "sticky tape sampling") and micro-chemical testing to detect the presence of proteins, with comparison against appropriate controls prepared from laboratory grade chemical samples, modern linen, a sample of $17^{\text {th }}$ century Spanish linen, heated linens, protein coated linens, etc. The chromophore accounting for the body image fibre colour would be a mixture of conjugated carbonyl structures generated within the cellulose polymer itself $[15$, 16]. The chemical investigations are in complete agreement with the image studies in concluding that the body images are not composed of applied pigment stains, or dyes and have been produced by a different process from that of the blood marks.

\section{Spectroscopic investigations}

X-ray Transmission Radiography. The water-stains, burned areas and the details of the weave were studied. The cloth weave were clearly seen and the water-stain margins showed up strongly.

X-ray Fluorescence, to provide estimates of elemental variations among selected areas of the cloth [1, 17]. Fairly high concentrations of Calcium were found over most of the cloth with lower concentration of Strontium. Calcium content is more or less uniformly distributed over the whole cloth, Iron distribution is uniform over the whole cloth with a few notable exceptions, both supporting the chemical conclusion that it derives from the manufacture of the linen from flax. No evidence appears for the trace minerals associated with mineralogical forms of Iron oxides (such as Cobalt or Nickel), neither evidence of applied pigment, within the range and sensitivity limits of the spectrometer [18].

X-ray Power Diffraction to identify compounds (phases) and the degree of creep (by study of the X-ray fibre pattern, so identifying fold marks) in the specimen. The adopted technique showed the presence of small quantities of a crystalline pigment material, such as hematite, on linen, but experimenters were unable to confirm the presence of large quantities of products from dried blood [18].

Electron Microprobe (Energy Dispersive Spectrometer, operating similarly to X-ray Fluorescence), in order to comparably identify elements present in the investigated samples. The iron oxide tested resulted "pure," yet testing of water-margin iron oxide does not really tell to a claim that artist's iron oxide resides in 'blood' areas.

Infrared Reflectance. The peak ratios of the carbonyl region to that of the hydroxyl one showed clear evidence of an increasing state of oxidation in going from the background cloth to body image to scorch to the burned areas. The typical amide absorptions associated with proteins could be evidenced in the blood mark spectra but not in those of the body images [9].

High Resolution Micro-Spectrophotometry investigation and Fourier Transform Infrared Spectroscopy (FTIR). Fibres 


\section{International Journal of New Technology and Research (IJNTR) ISSN:2454-4116, Volume-2, Issue-3, March 2016 Pages 86-95}

and particles extracted from the STURP sticky tape samples were examined and each type of fibre showed a distinctive absorption pattern. A conjugated carbonyl absorption pattern was clearly seen in the body image samples, but no evidence for protein amide bands, which, however, were clearly seen in the serum coated fibres and blood particles. Distinct bulk fibre chemical structures were identified. FTIR micro-studies were performed on a series of fibres removed from the STURP sticky tapes and on the samples employed in the radiocarbon dating [19-21]. These studies indicate at least three basic chemically distinct possible classifications:

- progressive oxidation: by monitoring the changes in the carbonyl intensity of the infrared spectra, a ranking of oxidation is possible; this supports an oxidation process for the image formation, explaining the water transfer of oxidation products.

- blood serum coated cellulose fibres and dried blood particles

- overall area specific bulk chemical differences in fibre cellulose.

These results confirmed and extended those obtained by Infrared Reflectance.

Colorimetry. The spectral reflection factors of a number of points of the Shroud have been measured through a portable spectrophotometer. A complete colour map of the Shroud was obtained through the Mobile Imaging Radiometer (MIR), with easy detection of any colour drift. The Turin Shroud appeared almost monochromatic and the chromaticity coordinates were placed in a very small region of the (x,y) plane [22].

Reflection ultraviolet-visible spectroscopy

Optical and scanning electron microscopy (SEM) investigation (reflection, transmission, phase contrast, fluorescence and polarization). The typical weight percent elemental composition patterns of the various TS's fibre types were assessed, evidencing a great deal of variability in the radiocarbon samples [23].

\section{E. Wet chemistry tests}

Wet chemistry tests (including enzymatic and immunochemical analysis) of materials removed from the surface of the Shroud. As a result, manganese, cobalt, nickel and aluminium could only be present at a level of less than $1 \%$. The water-margin iron oxide, hence, very probably was not an artist's iron oxide: 'very likely' since the minor possibility that an artist used pure haematite crystals to paint 'water-margins'.

\section{F. ${ }^{14}$ Carbon radio-dating}

${ }^{14}$ Carbon radio-dating carried out in the year 1988 in Arizona, Cambridge and Zurich on a unique specific sample of the cloth (split into several pieces) dated it in the period between A.D. 1260 and 1390 with 95\% confidence [24, 25]. No attention was devoted to: the possibility of contamination which might escape normal pre-treatment; the fact that Shroud had been through a church fire in A.D. 1532 and a series of other events that could conceivably affect its radiocarbon content by "carboxylation" or isotope exchange in a manner not yet understood or repeated under laboratory conditions; a possible thermal gradient that can be observed, with possible significant variations across the entire cloth; possible contamination due to mould, mildew and fungal growths, organic material such as bacterial or insect residues and fine particulates locked in the cellulose structure; the need of corrections into the intermolecular fractioning of carbon isotopes in plant cells, required for the more precise carbon dating of textiles made from plants (the ${ }^{14} \mathrm{C}$ and ${ }^{13} \mathrm{C}$ contents of cellulose, in fact, are different from those in other molecules and in the common vegetable biomass) [26]; alkylation of cellulose in an ageing fabric involves the direct participation of single-cell leavens from the surrounding environment: dissimilarities in the micro-organism populations of single geographical areas naturally influence the degree of alkylation of cellulose in fabrics from different parts of the world, and other motivations [26]. The possibility of an anomalous isotopic fractionation was also considered, which can be responsible of age's change [27]. Initial estimates of the kinetics constants for the loss of vanillin from lignin in both warp and weft threads taken from the centre of the radiocarbon sample prior to its distribution for dating, indicate a much older age for the cloth than the radiocarbon analyses.

Moreover, pyrolysis-mass-spectrometry results from the sample area coupled with microscopic and micro-chemical observations prove that the radiocarbon sample was not part of the original cloth of the Shroud [28].

\section{G. Immunofluorescence}

B. Bollone discovered by means of this method the presence of aloe and myrrh on the threads he took from the Shroud immediately after the exposition of 1978 [29].

\section{H. Forensic analysis}

Forensic analysis of the image has been carried out. Results are consistent with the chemical, microscopic and spectroscopic conclusion, including evidence for the elevated bilirubin levels indicative of trauma. Results are in agreement also with the computer analysis conclusions: the blood images penetrate the weave of the cloth, show cementation of fibres to one another and show capillary flow under the crossing threads of the weave.

A study was carried out on the structural features of the flax fibre in general and the possible role of lignin in image formation [30]. A technical product analysis of the cloth of the TS and its reproduction with similar characteristics was carried out, furthermore, using a part of the strip cut on 21 April 1988 for the radiocarbon dating [31]. Excimer laser irradiation experiments (emitted light spectrum in the ultraviolet and vacuum ultraviolet) of raw linen fabrics were recently carried out, looking for coloration comparable to that of the Sindonic Image. A very superficial Shroud-like coloration of linen yarns in a limited range of irradiation parameters was obtained, as well as dissimilar physical and photochemical processes accounting for both coloration and latent coloration, which may have been involved in the creation of the Sindonic Image [32].

Scientific discussions on physical, chemical, biological, forensic and historical aspects of the TS were recently carried out, especially making comparisons between the TS and possible copies [33]. Results, in particular, highlighted that: the total vacuum ultraviolet (VUV) radiation power required to colour a linen surface corresponding to a human body makes impracticable the reproduction of the whole TS 
image by using a single laser, since this power cannot be delivered by any VUV source built to date [34]; the TS image was certainly not produced by means that were available to a medieval forger or, very likely, by any kind of similar rubbing technique, since the technique itself seems unable to produce an image having the most critical TS image characteristics [35]. A list of evidences of the TS was defined, moreover, upon which to base further debates on the body image formation hypothesis, even if the TS characteristics are not definitive [36].

\section{FURTHER TESTING}

The following additional newer investigations were recommended by the STURP's Members:

- Biological assay for the micro-flora and micro-fauna

- Chemical structures

- Spectroscopic investigations, by using improved portable instruments with higher resolution and enhanced signal to noise ratios: spectral reflectance and fluorescence measurements can be extended to shorter wavelengths where organic and inorganic species have high and presumably distinguishable absorption.

- Fibre optic probes fluorescence

- Ultraviolet-visible emission at designated points of interest

- FTIR investigation at designated points of interest

- Advanced imaging techniques using filters

- Spectral imaging

- Surface pH measurements

- High Resolution Nuclear Magnetic Resonance (NMR). It allows distinguishing different chemical species, giving also structural information on spatial proximities of different resonant nuclei. For the TS studies, the NMR signals of ${ }^{1} \mathrm{H}$ and ${ }^{13} \mathrm{C}$ were suggested [37]. Polarized image overlay technique [38], useful in evaluating and confirming in the case of objects extremely closely resembling the images on the TS, such as the coins over the eyes and some of the floral images.

A new ${ }^{14}$ Carbon radio-dating, taking few grams of the cloth from three or more locations [39].

\section{FEASIBILITY OF NEUTRON BASED INVESTIGATION AND DISCUSSION}

Neutrons are very important tools for exploring and modification of materials' features at nano-scale and microscopic level. They have unique properties: charge neutrality and deep penetration, magnetic moment, large scattering cross section for light elements such as hydrogen and oxygen, sensitivity to isotopes as well as unique kinematics that allow simultaneous determination of position and motion of atomic, molecular or nano-scale structural units. High intensity neutron beams are produced at nuclear reactors or accelerator based spallation sources. It is often considered that access to NBT at such large scale facilities is difficult or cumbersome. In fact, this not the case, the major neutron source laboratories have a well-established access system and scientists performing NBT experiments are assisted by the local expert staff. Furthermore, a crucial advantage of neutrons in the domain of Cultural Heritage is the completely non-destructive nature of investigations. Two types of specific NBT are discussed below, relevant to the current subject. A description of the possible expected results for each NBT follows, together with an experimental section: the latter, reporting some results that show the reliability of the SANS method in studying linen textiles.

\section{A. Small Angle Neutron Scattering}

Small Angle Neutron Scattering (SANS) is a non-destructive technique, which gives an accurate and foremost complete characterisation of materials structures at micro- and nano-levels, providing statistically precise information averaged over a macroscopic volume. Parameters relative to the scattering objects (aggregates, pores, defects etc.), in particular, can be monitored, such as size, concentration, volume fraction and interface area. The theoretical bases of the SANS technique can be found in various references $[4,5,40-42]$. The same specimen can be studied or measured any number of times after either further usage or treatment. Fig. 1 shows a scheme of the SANS technique.

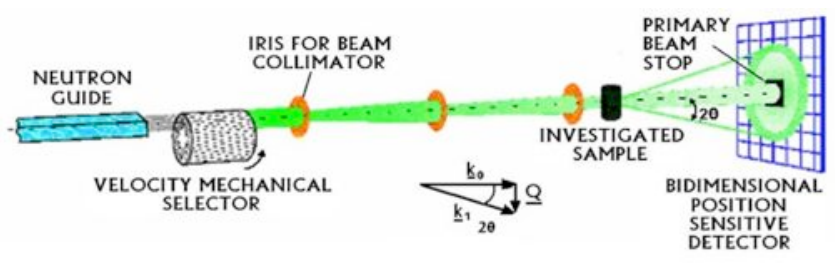

Fig. 1. Scheme of the SANS technique

A primary data treatment includes the regrouping of the two-dimensional scattering patterns depending on the type of scattering (isotropic or anisotropic), background subtraction, correction for transmission and normalization to standard samples, usually to $1 \mathrm{~mm}$ thick water sample. The scattered intensity at small angles is proportional to the square of the contrast, i.e. the difference between the scattering length densities of the so-called matrix and the nano-metric sized objects located inside the matrix [41, 43]. When the monochromatic plane wave illuminates the sample, scattered waves of variable intensities are emitted in all directions of space. The incident and scattered waves at an angle of $2 \theta$ can be described by their wave vectors $\bar{k}_{0}$ and $\bar{k}_{1}$ that defines the scattering vector $\bar{Q}$ :

$$
\bar{Q}=\overline{k_{1}}-\overline{k_{0}}
$$

In the case of elastic scattering:

$|Q|=\frac{4 \pi \sin \theta}{\lambda}$

The measured intensity at the detector can be expressed as:

$I(\lambda, \theta)=I_{0}(\lambda) \Delta \Omega \eta(\lambda) T V \frac{\partial \sigma}{\partial \Omega}(Q)$ 
where $I_{0}$ is the incident flux, $\eta$ is the detector efficiency, $T$ is the sample transmission, $V$ is the sample volume and $(d \sigma /$ $d \Omega(Q)$ is a function known as the microscopic differential cross-section. $(d \sigma / d \Omega)(Q)$ possesses the dimension of (length) ${ }^{-1}$ and it is usually expressed in units of $\mathrm{cm}^{-1}$. A Guinier-fit is made subsequent to a proper calibration and radial averaging. In the Guinier-region, the expression:

$$
I(\lambda, \theta)=\operatorname{const}(\lambda) \exp \left(-Q^{2} R_{g}^{2} / 3\right)
$$

which describes the behaviour of the scattering intensity.

In (4), $R_{g}$ is the average radius of gyration and $I$ the measured intensity, containing the different constant values characteristic of the instrumentation and the analysed sample such as transmission, detector pixel size and sample volume. The Guinier model allows determining the gyration radius (that gives information on the scattering objects) and the measured intensity values versus $Q\left(\AA^{-1}\right)$.

Linen fibres are an extremely stiff material in the longitudinal direction of the fibre; the macromolecules are generally oriented in the same direction. Since they are plant cell walls, they can be considered as composites made of cellulose nano-crystals (micro-fibrils) embedded in a disordered matrix. A SANS analysis of the considered linen fibres could probe the atomic- and nano-scale structure and magnetic order (if any) under diverse conditions, giving the following information on their multilevel and complicated morphology:

- sizes of dense (crystalline) and rare (amorphous) nano-scale regions

- their arrangement (possible periodicity) along the fibres and radial direction

- degradation degree of the cellulose fibrils

- molecular package densities in amorphous and crystalline domains using the cross sections in absolute units

- anisotropy in structural elements orientation as dependent on the methods of production (modification)

- existence of several structural levels (domains, filaments, lamellar structures etc.) at scales from $1 \mathrm{~nm}$ up to few microns

- if it will be allowed to use the wetting in heavy and light water, the different swelling of crystalline and amorphous regions and its influence on the fibres' morphology after their drying

- structure and amount of defects (e.g. nano-pores, nano-cracks), showing the ageing processes as dependent on the date (century), the methods of production, macroscopic parameters of the samples (length and diameter of fibres)

structure of the fibres' surface (nano-defects; fractal character of surface, i.e. the quality of the border which can be perfect or very rough).

\section{B. Neutron Diffraction}

Neutron Diffraction (ND) allows studying the structural properties of an extensive range of materials such as fibrous polymers, biopolymers, metals, fibres, stones and marbles, supplying information on crystal and magnetic structure and micro-strains phase composition [4]. The microstructure is normally associated with the material properties and the manufacturing process. ND can be used also to study:
- the molecular structure

- the hydrogen bonding system and arrangement, providing the coordinates of all atoms in the investigated material

- texture, obtaining information on the disturbed grain orientation in a specimen body [44].

X-ray diffraction and ND have already shown their complementarity in a variety of cases, e.g. see [45]. Among the various complementarities, hydrogen scattering can be highlighted: it is rather weak for X-rays, while it is strong for neutrons. This characteristic is even more marked concerning deuterium. Deuterated fibres, in particular, are able to diffract neutrons with intensities that are significantly dissimilar from those diffracted from hydrogenated fibres. This allows ND to locate ordered water in fibres of hydrated biological polymers. The substitution of $\mathrm{H}_{2} \mathrm{O}$ by $\mathrm{D}_{2} \mathrm{O}$ in the fibre can be exploited to enhance the scattering power of the water molecules, allowing us to determine the position of hydrogen atoms involved in hydrogen bonding. Samples can be studied, thus, either in hydrogenated or in hydrogen-deuterium exchanged deuterated form. Mercerized fibres can be also considered, to be investigated in a hydrogenated form (employing, e.g., $\mathrm{NaOH}: \mathrm{H}_{2} \mathrm{O}$ as mercerizing medium) and in a deuterated form (employing, e.g., $\mathrm{NaOD}: \mathrm{D}_{2} \mathrm{O}$ ). Data can be compared to similar parameters obtained for natural, synthetic and mineral (e.g. basaltic wool, which is widely used) modern fibres. Preliminary tests would be carried out, thus, on modern linen specimens as well as samples belonging to different ages (e.g., Middle Ages). ND data treatments are carried out following particular procedures (e.g., quantitative Rietveld analysis in order to identify phases, quantitative assessment of the mineral composition and lattice constant determination).

\section{Instrumentations and experimental section}

SANS and ND experiments are proposed to carry out at the BNC operating the $10 \mathrm{MW}$ Research Reactor (BRR), which is Hungary's foremost scientific research and development suite, focused on delivering the benefits of nuclear science to the Hungarian and European research community, including the Cultural Heritage sector [46]. In the current study, SANS and ND are the most relevant techniques to be applied.

\section{1) The SANS instrument}

The neutron wavelength of the SANS spectrometer of the BRR can be varied in the range 3-25 $\AA$ and a multidisc type velocity selector can provide the choice of a given wavelengths, the wavelength spread being adjustable in the range $12-30 \%$. The neutron flux at the guide exit is $5 \times 10^{7}$ $\mathrm{n} / \mathrm{cm}^{2} \mathrm{~s}$. A collimator system enables us to adjust the beam divergence (via supermirrors guides and proper diaphragm sets) of incoming neutrons to the investigated object, where

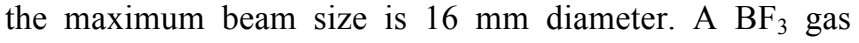
filled, two dimensional position sensitive $64 \times 64$ pixels multi-detector counts the scattered neutrons. The pixel size is $1 \mathrm{~cm} \times 1 \mathrm{~cm}$. The scattered angle covers a momentum transfer $q$ range of $0.003-0.5 \AA^{-1}$, allowing density, composition and magnetization fluctuations in materials under test to be measured on a length scale in the range 
10-1000 A. Fig. 2 shows the scheme of the SANS spectrometer of the BRR.

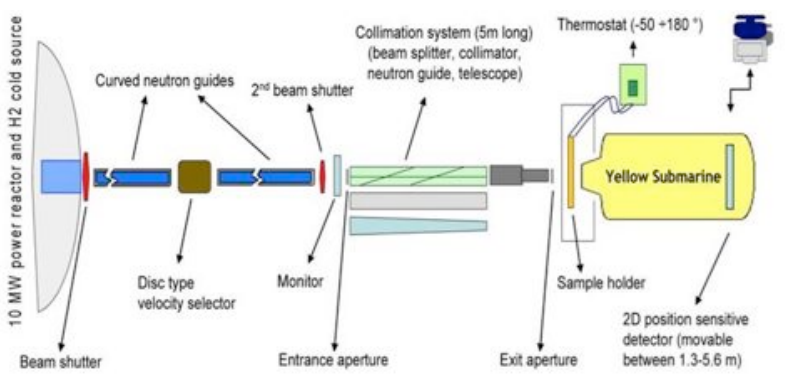

Fig. 2. SANS facility of the BNC

The sample environment includes a thermo-stated sample changer, electromagnet, cryostat, furnace and other specific devices.

\section{2) The ND-TOF instrument}

This is a high-resolution time-of-flight (TOF) powder diffractometer installed on a thermal neutron beam in a separate guide-hall. Its monochromator consists of a 5-chopper system with supermirror neutron guides. This is a highly demanded instrument by users for various research programmes such as structural analysis of multiphase alloys, strain analysis in structural materials, identification of archaeological objects. Fig. 3 shows the scheme of the TOF instrument at the BNC, while Fig. 4 shows an illustration of the measuring range and the resolution of the same instrument.

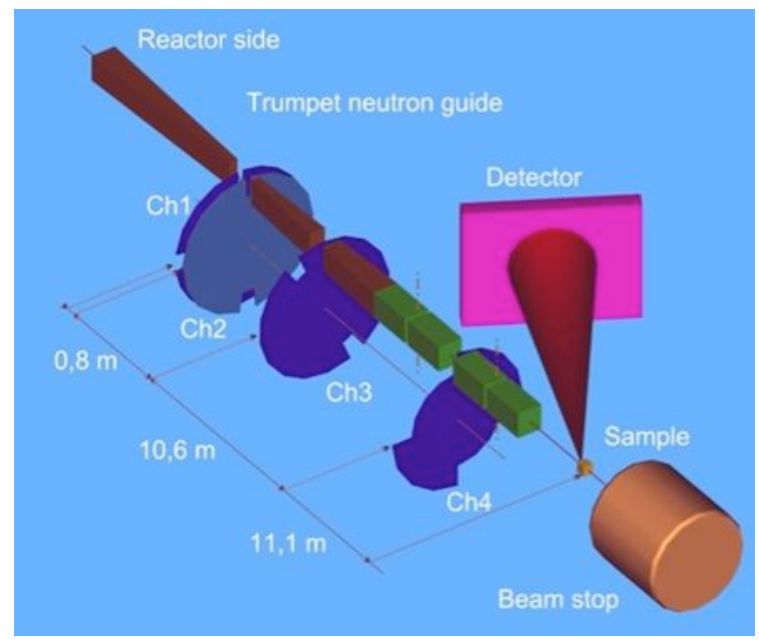

Fig. 3. Scheme of the TOF instrument at the BNC

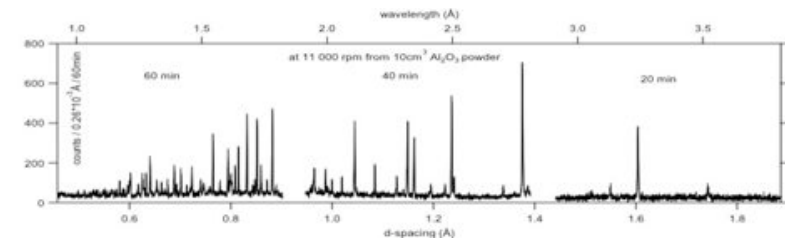

Fig. 4. Illustration of the measuring range and the resolution of the TOF instrument at the BNC: diffractogram from a sintered $\mathrm{Al}_{2} \mathrm{O}_{3}$ sample.

A recent typical cultural heritage highlight: The structure and chemical composition of "mankind's earliest iron tool" from a London collection was recently studied by TOF diffraction, PGAA and neutron tomography. The meteoritic origin was confirmed and it was first discovered that this 5500 years old "iron pearl" was made by wrapping of a metal sheet [47].

\section{3) Experimental section}

Various experimental analyses have been carried out in order to verify the reliability of the SANS method in studying textiles. Below we give a typical example of investigations on textile fibres performed at the BNC SANS spectrometer. Textile fibres typically consists of the elements H:C:N:O (other). For example wool and animal hair at 5:38:22:21:14, whereas vegetable based textile fibres consist typically of $11: 4: 1: 84: 0$ and synthetic fibres 6:51:11:32:0 respectively. The $14 \%$ other of wool is in the form of $\mathrm{S}, \mathrm{Cl}$ and some trace elements, which plays an important role in the quality of the textile fibre, especially after chemical treatment or "history" of the textile.

The quality of chemically treated fibres strongly depends on their wetting properties. These fibres are modified by the location of chemically active elements contributing to their structural properties of natural and artificial origin at different water contains. Various chemical treatment processes are applied to fibre pre- and post woven. Analysis of the quality of the product, in terms of strength, texture, flexibility, improved hydrophilic/hydrophobic and/or oleophobic properties are usually undertaken through internationally accorded testing procedures. The process is empirical, in that, there is a lack of understanding of the underlying material modification at the atomic/molecular level. In the case of wool, for example, the wetting properties linked to proteins consisting from fibres the conformation due to wetting process can be monitored. The SANS measurement showed the details of these process [48]. For wetting, heavy water is added providing high contrast and, showing the surface properties of fibres, too. Experiment carried out at the SANS spectrometer of the BRR showed a significant difference between the treated and untreated samples.

Fig. 5 shows the picture of a wool fibre obtained by SEM, while Fig. 6 and Fig. 7 show the spectra (isointensity contours) obtained by SANS from natural wool fibres that exhibit a very low or very high optical lustre.

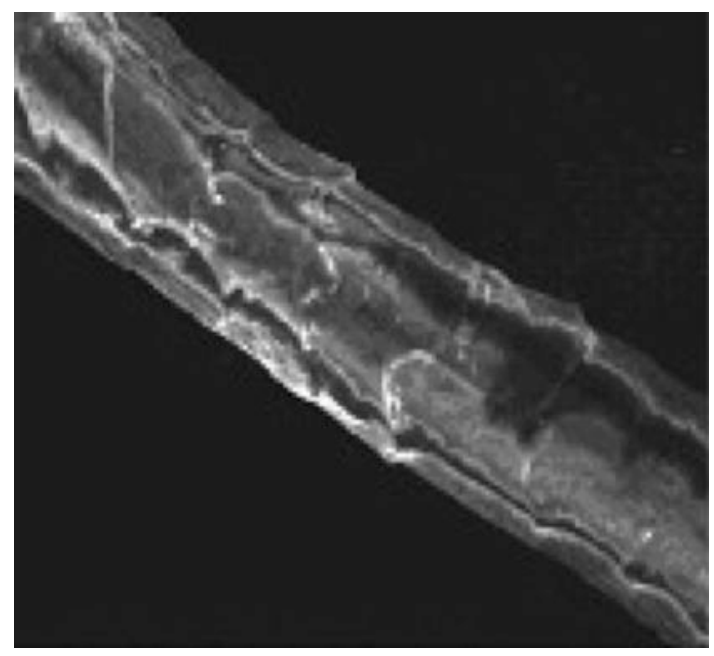

Fig. 5. SEM picture of a wool fibre 


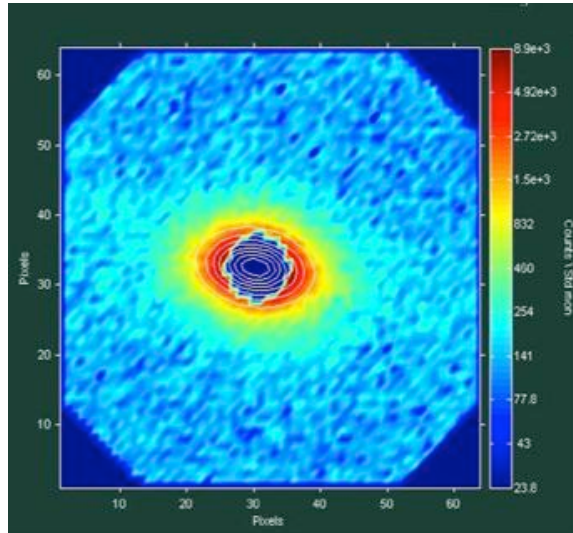

Fig. 6. SANS spectrum from dull wool

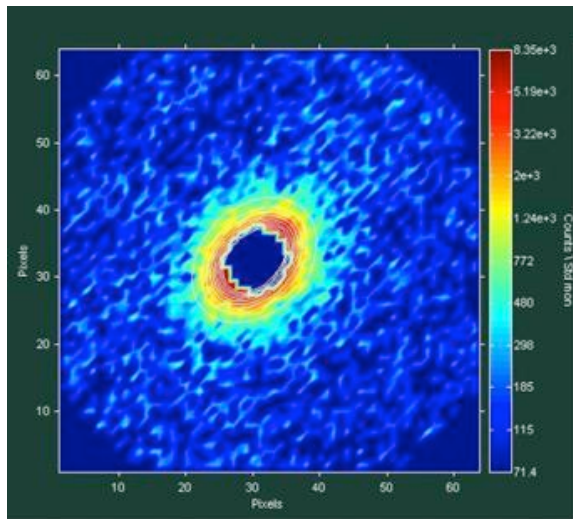

Fig. 7. SANS spectrum from lustrous wool

Different anisotropy of neutron scattering properties for the different types of fibre was observed. Analysing the obtained spectra a difference of two type of building element, an isotropic and an elongated component with elongation ratio $1: 1.5$ could be obtained.

Experimental analyses of several samples of new and aged linen fabrics of known age and characteristics have been recently carried out, in order to verify the reliability of the SANS method for this kind of fibres [49]. 9 linen fabric samples dated from Old Kingdom to Ptolemaic ages (2200-300 B.C.) were investigated, as well as 5 modern linen fabric samples, added for comparison. Fig. 8 shows two fragments of one of the investigated ancient samples.

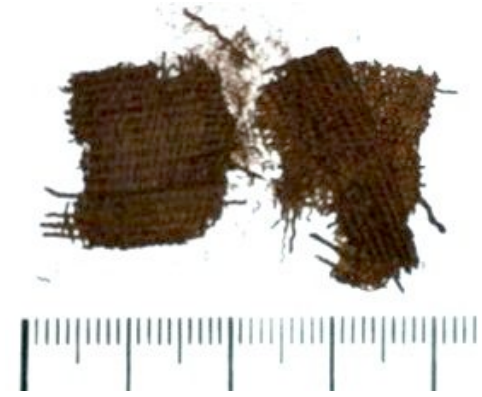

Fig. 8. Two fragments of ancient Gebelein linen fabric, period 3500-2000 A.C., tabby weave and warp-faced, $26-28 / \mathrm{cm}$ warp count, $9 / 10 / \mathrm{cm}$ weft count, investigated by SANS

SANS allowed determining the nanoscaled differences between the ancient and modern fabrics, and some results are reported as follows.
A good small angle scattering signal resulted in case of all (ancient and modern) specimens, and this effect can be attributed to the scattering from the surface of the nanofibrils and microfibers. Concerning the ancient fabrics, no orientation was observed on the scattering pictures: this could be due to the samples' degradation and the damage of the cellulose fibrils. The modern samples exhibited anisotropy along the linen wire axis, and an oriented scattering was observed in two perpendicular directions, comparable to the fabric's weaving. The samples have been treated as they would be isotropic scatterers, since the two diverse orientations could not be well-separated, and the scattering was not coming from the cross section of the linen nanofibrils, but from their length, which was outside of the observation range of the instrument. This allowed to obtain fractal exponents for the modern fabrics as well. As a first approximation to the subject, ancient and modern linen samples have been compared, which showed different fractal like behaviour. The modern samples fractal exponents were characteristic to the volume fractals $(2<p<$ $3)$, while the ancient samples showed surface fractal behaviour $(3<p<4)$. The fractal behaviour of the linen fibre can be associated with its hierarchical structure and its heat-retain which is the reason of its use as clothing tool, starting even from the ancient times [50]. In the case of the ancient linen, the surface fractal behaviour is explained by the breaking of the hydrogen bonds between the cellulose units of the long polymer chains: the fibres became homogenous, and hierarchically non-structured. The presence of the anisotropy for the modern samples creates reference to the existence of whole - not destroyed - fibrils in the fabric, i.e. a good indicator of their modern origin. The disappearance of volume fractality and anisotropy for the ancient samples agrees with results obtained by other techniques. SANS-data for all the groups of the samples were treated using the models of surface and mass fractals. In both cases the scattering intensities distributions $I(q)$ in the experimental range of momentum transfer $q$ obey the exponential functions (Fig. 9) with addition of incoherent background :

$I(q)=A / q^{D}+B$

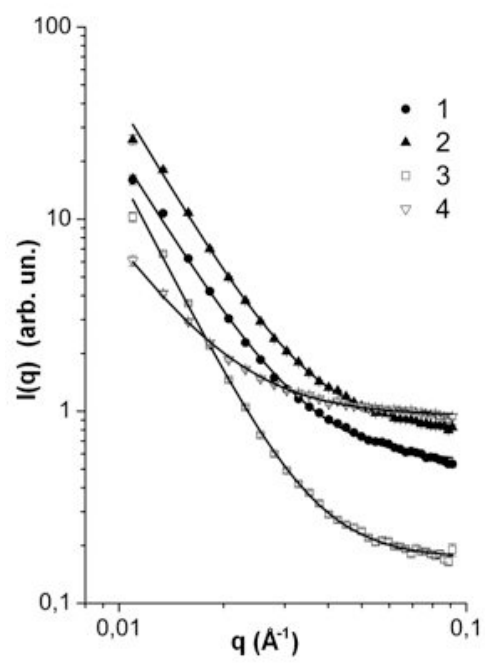

Fig. 9. Scattering intensities for ancient samples dating in the period 2590-2180 B.C. Lines are fitting functions (1) 
Here the first term shows coherent scattering from the linen fibres. The coefficient $A$ is proportional the contract factor of fibre substance regarding to the surrounding air and the volume content of substance in the sample. The constant additive $B$ is defined mainly by the amount of hydrogen in the composition of linen, since the protons have a great incoherent scattering cross section. The magnitude of $B$ normalized to the mass of the sample, hence, shows the hydrogen concentration in molecular substance of linen and some possible changes in hydrogen content during several thousands of years (aging effects). The exponent $D$ is a crucial parameter which indicates really subtle structural features of scattering objects. Various mass fractals have the exponent within the interval, $1 \leq D<3$, while surface fractals are characterized by larger parameter, i.e. $3<D \leq 4$. The issued fractal dimension of the surface $D_{S}=6$ - D alters within the limits, $2 \leq D_{S}<3$. The lower limit $D_{S}=2$ corresponds to a sharp surface, but the upper limit $D_{S} \rightarrow 3$ indicates a very defective and highly developed surface. As it has been found (Fig. 10), the modern samples have shown low exponent $D=2.6-2.9<3$. Thus these modern materials are composed of very thin (molecular sized) filaments very branched (entangled), and substantial free volume between them should exist.

On the other hand, the ancient materials (age 2600-3500 years) have demonstrated well defined structural units being dense fibres with revealed borders, $D \sim 3.1-3.6$. The surface of these fibres has a fractal dimension $D_{S}=6-D=2.4-2.9$ which comes up with the increase of the age of material. For the oldest samples (4500 years) the exponent $D$ resulted close to the limit $D \rightarrow 3$. The aging process, thus, is revealed in a degradation of fibres' surface which become very defective and large. Even for the oldest linen fabric, nevertheless, it was observed that the structure is very different as compared to this one for modern linen material. The latter is a composite of very thin (few nanometers in diameter) filaments branched and entangled that is clear visible from the magnitudes of the exponents $D=2.6-2.9$ (Fig. 10).

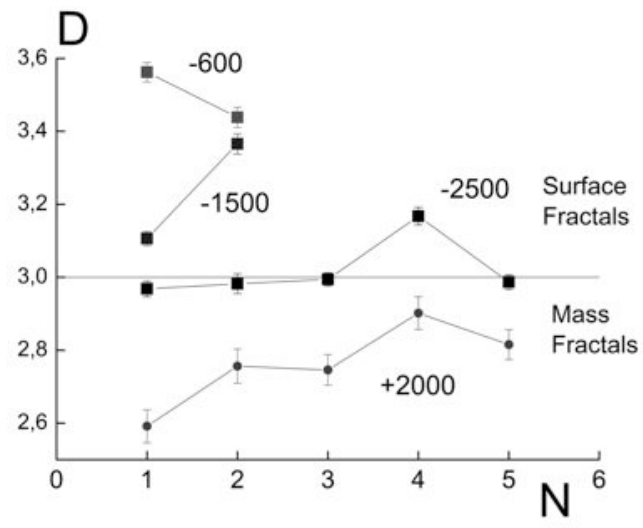

Fig. 10. The magnitudes of the exponents $D(N)$ vs. the numbers of the samples of materials. Evolution of structural properties of linen materials vs. their age.

Finally the evolution of the nano-scale structural properties of the linen is presented in Fig. 11.

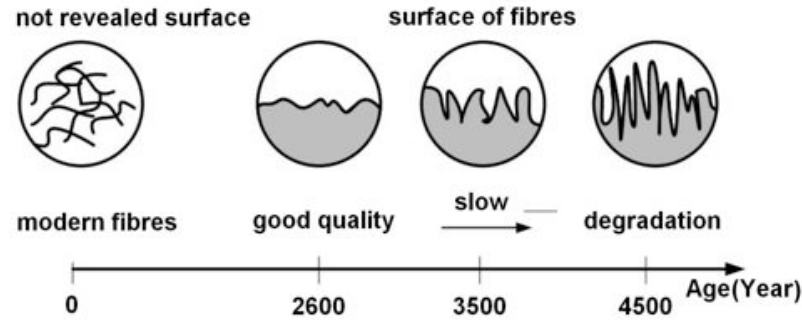

Fig. 11. Evolution of structural properties of linen materials vs. their age.

In a successive data treatment, the values of the parameter $D$ were averaged in each samples' series, allowing us to observe structural changes that can be interpreted as follows: in the ancient materials, after an induction period $\left(t^{*} \approx 1350\right.$ Year) a degradation process initiates, and the parameter $D(t)$ shows an exponential decay described by the function:

$D(t)=\left(D_{0}-D_{\infty}\right) \exp \left[-\left(t-t^{*}\right) / \tau\right]+D_{\infty}$

where $D_{0}=4$ is the initial magnitude of the fractal exponent for completely sharp border, $D_{\infty} \approx 2.6$ is a final fractal exponent in the limit of times much longer than a characteristic time of degradation $\tau \approx 2.55 \cdot 10^{3}$ Year. It should be stressed that the exponent $D_{m} \approx 2.8$ related to the modern linen fabrics is close to the limit $D(t \rightarrow \infty)=D_{\infty} \approx 2.6$ for old linen materials.

A final remark is due, related to the sampling. NBT have been presented as non-destructive, thus any neutron investigation of the TS would be without any harm on it. It is however, not realistic to consider that the TS could be moved to a neutron source facility, thus sampling will be necessary in order to analyse the linen fibres. Number of samples and sample size for obtaining credible results is a vital knowledge when considering analysing a unique object as the TS. If for example, new ${ }^{14}$ Carbon radio-dating experiments are to be performed, taking few grams of the cloth from three or more locations, the same samples can be used for NBT investigations - and this is crucial to understand that these samples will not suffer any hard, thus they are intact for any further studies of even to restore into the original place on the Shroud.

\section{CONCLUSION}

The results obtained by adopting NBT in materials characterization have often proved their strong support for better understanding of material's characteristics and behaviour. SANS has proved itself as a highly helpful tool to investigate micro- and nano-structural aggregates, defects, pores in various types of materials and it represents, in the present case, a valuable advanced method for contributing to the knowledge of various parameters, properties and other important characteristics of the considered material. ND is capable to supply information on the structural properties and, in this case, it would allow completing the data obtained by SANS in investigating the molecular structure, the hydrogen bonding system and the texture of the relevant textiles (the Shroud and reference materials). Both neutron techniques would provide 


\section{International Journal of New Technology and Research (IJNTR) ISSN:2454-4116, Volume-2, Issue-3, March 2016 Pages 86-95}

substantial new data to complement the analytical and crystallographic information, which are crucial to comprehend the structural basis for the chemical, physical, and biological properties of the considered material. In particular, SANS-studies of very old linen materials have shown their very different structural features at nano-scales comparatively to modern materials. Is was established that old linen are composed of fibres with good revealed borders which demonstrate slow degradation due to defects' formation at characteristic times $\tau \approx 2.5 \cdot 10^{3}$ Year after induction period $t^{*} \approx 1350$ Year. On the other hand, modern materials displayed alternative structures which are of mass-fractal type. Such structures are composed of very thin filaments (few nanometers in diameter) very branched and (or) entangled. In these new materials there were not observed any sharp borders of fibres. As it was shown by the data extrapolation to long times, the old materials may get a rare mass-fractal structure only in the period $t \geq 10^{4}$ Year. This is a real evidence of the possible contribution to the understanding of the TS origin. In simple words, the nano-scale structure of linen materials revealed by small angel neutron scattering, can be well distinguished on one hand, for modern textiles of thin filaments with rather porous nature in the bulk and on the other hand, old linen materials, where the aging degradation due to molecular level defects leads to shrinking of pores, thus getting a more dense structure. This transformation from a lower density of fresh material to a less porous nano-structure in old linens can be used as a measure of aging process, thus support dating information. The key point of the proposed current neutron study - as a first approach by this powerful technique with respect to the TS - is that all hypotheses will be able to be tested by reproducible experiments. The non-invasive nature of these measurements is essential to assure the repeatability and reproducibility of the measurements. One should consider in choosing the most appropriate samples for the neutron investigations and - in this way - in carrying out the proper measurements, random and/or systematic errors can be avoided. As shown by the relevant examples above a set of neutron experiments would be able to provide original information in completing the multifaceted mosaic to understand the enigmas of the Sindonic Image, as well as to contribute in preserving and sustaining this great heritage for present and future generations. Finally, the described methods can be implemented in new studies, not only of the TS, but also for various other ancient textiles.

\section{ACKNOWLEDGMENT}

Prof. Bruno Barberis, Director of the International Centre of Sindonology, is particularly acknowledged for having kindly supplied valuable information. Prof. Peter Wadhams is acknowledged for useful discussions.

\section{REFERENCES}

[1] The Turin Shroud: past, present and future, Proc. Int. Scientific Symposium, Turin, 2-5 March 2000, S. Scannerini and P. Savarino, Eds., Turin, Italy, Sindon - Centro Internazionale di Sindologia, 2000.

[2] F. Barbesino and M. Moroni, "Effects of neutron irradiation on linen fibres and consequences for a radiocarbon dating", in Proc. Int. Conf. on The Shroud of Turin: Perspective on a Multifaceted Enigma, Columbus, Ohio, August 14-17, 2008. Available: http://www.ohioshroudconference.com/papers/p03.pdf.

[3] J. Lenz, J. Schurz and E. Wrentschur. (1988). The length of the crystalline domains in fibres of regenerated Cellulose. Determination of the crystallite length of Cellulose II by means of Wide-Angle X-Ray Diffraction and Transmission Electron Microscopy. Holzforschung. 42(2). pp. 117-122.

[4] M. Rogante, "Applicazioni Industriali delle Tecniche Neutroniche", in Applicazioni Industriali delle Tecniche Neutroniche $\AA$, Proc. 1st Italian Workshop for Industry, M. Rogante, Ed., Civitanova Marche, Italy, Rogante Engineering, 2008, pp. 40-120.

[5] M. Rogante and L. Rosta, "Nanoscale characterisation by SANS and residual stresses determination by neutron diffraction related to materials and components of technological interest", in Proc. SPIE 5824, W. J. Blau, D. Kennedy and J. Colreavy, Eds., Bellingham, WA, SPIE, 2005, pp. 294-305.

[6] Sindone: cento anni di ricerca, B. Barberis and G. M. Zaccone, Eds., Rome, Istituto Poligrafico Zecca dello Stato, 1998.

[7] A. D. Adler, R. Selzer and F. Deblase, "Further spectroscopic investigations of samples of the Shroud of Turin", in The Orphaned Manuscript - A Gathering of Publications on the Shroud, A. D. Adler, Ed., Turin, Italy, Effatà, 2002, pp. 93-102.

[8] V. D. Miller and S. F. Pellicori. (1981). Ultraviolet fluorescence photography of the Shroud of Turin. Journal of Biological Photography. 49. pp. 71-85.

[9] J. S. Accetta and J. S. Baumgart. (1980). Infrared Reflectance Spectroscopy and Thermographic Investigations of the Shroud of Turin. Applied Optics. 19(12). pp. 1921-1929.

[10] S. F. Pellicori. (1980). Optical Properties of the Shroud of Turin. Applied Optics. 19(12). pp. 1913-1920.

[11] R. Gilbert and M. M. Gilbert. (1980). Ultraviolet-visible reflectance and fluorescence spectra of the Shroud of Turin. Applied Optics. 19(12). pp. 1930-1936.

[12] R. W. Mottern, R. J. London and R. A. Morris (1980). Radiographic Examination of the Shroud of Turin - a Preliminary Report. Materials Evaluation. 38. pp. 39-44.

[13] S. Pellicori, "Spectrophotometry of the Shroud: a critical review", in The Turin Shroud: past, present and future. Proc. Int. Scientific Symposium, Turin, 2-5 March 2000, S. Scannerini and P. Savarino, Eds., Turin, Italy, Sindon - Centro Internazionale di Sindologia, 2000, pp. 101-110.

[14] N. Balossino, "Computer processing of the body image", in The Turin Shroud: past, present and future. Proc. Int. Scientific Symposium, Turin, 2-5 March 2000, S. Scannerini and P. Savarino, Eds., Turin, Italy, Sindon - Centro Internazionale di Sindologia, 2000, pp. 111-124.

[15] J. Heller and A. Adler. (1981). A Chemical Investigation of the Shroud of Turin. Canadian Forensic Society Scientific Journal. 14(3). pp. 81-103.

[16] J. Jumper, A. Adler, J. Jackson, S. Pellicori, J. Heller and J. Druzic, "A Comprehensive Examination of the Various Stains and Images on the Shroud of Turin", In Archaeological Chemistry III, ACS Advances in Chemistry Series 205, Chapter 22, J. B. Lambert, Ed. Washington: American Chemical Society, 1984, pp. 447-476.

[17] R. A. Morris, L. A. Schwalbe and J. R. London. (1980). X-Ray Fluorescence Investigation of the Shroud of Turin. X-Ray Spectrometry. 9. pp. 42-47.

[18] R. Jenkins, "X-ray radiography and fluorescence on the Shroud", in The Turin Shroud: past, present and future. Proc. Int. Scientific Symposium, Turin, 2-5 March 2000, S. Scannerini and P. Savarino, Eds., Turin, Italy, Sindon - Centro Internazionale di Sindologia, 2000, pp. $75-88$

[19] N. Deblase, "Characterization of fibers by Fourier transform IR microspectroscopy", in The Turin Shroud: past, present and future. Proc. Int. Scientific Symposium, Turin, 2-5 March 2000, S. Scannerini and P. Savarino, Eds., Turin, Italy, Sindon - Centro Internazionale di Sindologia, 2000, pp. 265-272.

[20] A. D. Adler, "Updating Recent Studies on the Shroud of Turin", in Archaeological Chemistry: Organic, Inorganic and Biochemical Analysis, ACS Symposium Series 625, M. V. Orna, Ed., Washington: American Chemical Society, 1996, ch. 17, pp. 223-228.

[21] Atti del III Congresso Internazionale di Studi sulla Sindone. P. L. Baima Bollone, M. Lazzaro and C. Marino, Eds., Turin, Italy: The International Centre of Sindonology, 1998.

[22] P. Soardo, "Colourimetry of the Shroud", in The Turin Shroud: past, present and future. Proc. Int. Scientific Symposium, Turin, 2-5 March 2000, S. Scannerini and P. Savarino, Eds., Turin, Italy, Sindon Centro Internazionale di Sindologia, 2000, pp. 89-100.

[23] A. D. Adler, R. Selzer and F. Deblase, "Further spectroscopic investigations of samples of the Shroud of Turin", in The Orphaned 
Manuscript - A Gathering of Publications on the Shroud, A. D. Adler, Ed., Turin, Italy, Effatà, 2002, pp. 93-102.

[24] R. Otlet and J. Evin, "The present state of radiocarbon dating", in The Turin Shroud: past, present and future. Proc. Int. Scientific Symposium, Turin, 2-5 March 2000, S. Scannerini and P. Savarino, Eds., Turin, Italy, Sindon - Centro Internazionale di Sindologia, 2000, pp. $455-478$.

[25] P. E. Damon, D. J. Donahue, B. H. Gore et al. (1989). Radiocarbon dating of the Shroud of Turin. Nature. 337. pp. 611-615.

[26] A. Ivanov, "Carbon dating of the Turin Shroud: reasons for scepticism, alternative approaches, prospects and further research", in The Turin Shroud: past, present and future. Proc. Int. Scientific Symposium, Turin, 2-5 March 2000, S. Scannerini and P. Savarino, Eds., Turin, Italy, Sindon - Centro Internazionale di Sindologia, 2000, pp. 479-494.

[27] Y. Saillard, "Isotopic fractionation required for explanation of Turin Shroud's age by contamination during 1532 fire", in The Turin Shroud: past, present and future. Proc. Int. Scientific Symposium, Turin, 2-5 March 2000, S. Scannerini and P. Savarino, Eds., Turin, Italy, Sindon - Centro Internazionale di Sindologia, 2000, pp 523-530.

[28] R. R. Rogers. (2005). Studies on the radiocarbon sample from the Shroud of Turin. Thermochimica Acta. 425(1-2). pp. 189-194.

[29] S. Rodante, "The genesis of the Shroud imprints: experimental studies", in The Turin Shroud: past, present and future. Proc. Int. Scientific Symposium, Turin, 2-5 March 2000, S. Scannerini and P. Savarino, Eds., Turin, Italy, Sindon - Centro Internazionale di Sindologia, 2000, pp. 167-168.

[30] J. Cardamone, "Structural features on the flax fiber in general and the possible role of lignin in image formation", in The Turin Shroud: past, present and future. Proc. Int. Scientific Symposium, Turin, 2-5 March 2000, S. Scannerini and P. Savarino, Eds., Turin, Italy, Sindon Centro Internazionale di Sindologia, 2000, pp. 143-148.

[31] P. Vercelli, "The cloth of the Holy Shroud: a technical product analysis of the cloth and its reproduction with similar characteristics", in The Turin Shroud: past, present and future. Proc. Int. Scientific Symposium, Turin, 2-5 March 2000, S. Scannerini and P. Savarino, Eds., Turin, Italy, Sindon - Centro Internazionale di Sindologia, 2000, pp. $169-176$

[32] P. Di Lazzaro, D. Murra, A. Santoni, E. Nichelatti and G. Baldacchini, Colorazione simil-sindonica di tessuti di lino tramite radiazione nel lontano ultravioletto. Report RT/2011/14/ENEA. Frascati, Rome: ENEA UTFUS-DITE, 2011.

[33] Proc. Int. Workshop on the Scientific Approach to the Acheiropoietos images, P. Di Lazzaro, Ed., Rome: IWSAI, ENEA, 2010.

[34] P. Di Lazzaro, D. Murra, A. Santoni and G. Baldacchini, "Sub-micrometer coloration depth of linens by vacuum ultraviolet radiation", in Proc. Int. Workshop on the Scientific Approach to the Acheiropoietos images, P. Di Lazzaro, Ed., Rome: IWSAI, ENEA, 2010, pp. 3-10.

[35] T. Heimburger and G. Fanti, "Scientific comparison between the Turin Shroud and the first handmade whole copy", in Proc. Int. Workshop on the Scientific Approach to the Acheiropoietos images, $\mathrm{P}$. Di Lazzaro, Ed., Rome: IWSAI, ENEA, 2010, pp. 19-27.

[36] G., Fanti, J. A. Botella, F. Crosilla, F. Lattarulo, N. Svensson, R Schneider et al., "List of evidences of the Turin Shroud", in Proc. Int. Workshop on the Scientific Approach to the Acheiropoietos images, $\mathrm{P}$. Di Lazzaro, Ed., Rome: IWSAI, ENEA, 2010, pp. 67-75.

[37] J. Virlet, "The Shroud literature and the ${ }^{14} \mathrm{C}$ datation: solid state NMR, a useful tool", in The Turin Shroud: past, present and future. Proc. Int. Scientific Symposium, Turin, 2-5 March 2000, S. Scannerini and P. Savarino, Eds., Turin, Italy, Sindon - Centro Internazionale di Sindologia, 2000, pp. 177-188.

[38] A. Whanger, "Evidence for radiation from the Shroud image itself in the formation of the Shroud images", in The Turin Shroud: past, present and future. Proc. Int. Scientific Symposium, Turin, 2-5 March 2000, S. Scannerini and P. Savarino, Eds., Turin, Italy, Sindon Centro Internazionale di Sindologia, 2000, pp. 189-208

[39] W. Meacham, "Thoughts on the Shroud ${ }^{14} \mathrm{C}$ debate", in The Turin Shroud: past, present and future. Proc. Int. Scientific Symposium, Turin, 2-5 March 2000, S. Scannerini and P. Savarino, Eds., Turin, Italy, Sindon - Centro Internazionale di Sindologia, 2000, pp. 441-454.

[40] O. Glatter and O. Kratky. Small Angle X-ray Scattering. Academic Press, London, 1982.

[41] C. Williams, R. P. May and A. Guinier, "Small-Angle Scattering of X-rays and Neutrons", in Characterisation of Materials, Vol. 2B of Materials Science and Technology, E. Lifshin, Ed. Weinheim, Germany: VCH Verlagsgesellschaft, 1994, pp. 611-656.
[42] Rietveld, H. M. 1969. A profile refinement method for nuclear and magnetic structures. Journal of Applied Crystallography, 2. 65-71.

[43] H. M. Rietveld (1967). Line profiles of neutron powder diffraction peaks for structure refinement. Acta Crystallographica. 22. pp. $151-152$.

[44] M. Rogante. (2001). Tessitura e proprietà dei materiali. Progettare. 247. pp. $72-76$.

[45] M. Rogante, P. Mikula and M. Vrána. (2011). Residual stresses assessment in coated materials: complementarity between Neutron and X-ray techniques. Key Engineering Materials. 465. pp. 259-262.

[46] Zs. Kasztovszky and L. Rosta. (2012). How can Neutrons Contribute to Cultural Heritage Research?. Neutron News. 23. pp. 25-28.

[47] T. Rehren, T. Belgya, A. Jambon, G. Kali, Zs. Kasztovszky, Z. Kis et al. (2013). 5,000 years old Egyptian iron beads made from hammered meteoritic iron. Journal of Archaeological Science. 40(12). pp. 4785-4792.

[48] Q. S. Ye, S. Borbely and G. Horvai. (1999). Microstructure of ion selective plasticized PVC membranes studied by small angle neutron scattering. Analytical Chemistry. 71. pp. 4313-4320.

[49] M. Rogante, M. Borla, I. Kovács, V. T. Lebedev, A. Len, D. Picchi, L. Rosta and Z. Szökefalvi-Nagy, "SANS and PIXE investigation of ancient linen fabrics dated from Old Kingdom to Ptolemaic ages (2200-300 B.C.)", to be published.

[50] J. Fan, J. Liu and J. He. (2008). Hierarchy of wool fibers and fractal dimensions. International Journal of Nonlinear Sciences and Numerical Simulation. 9(3). pp. 293-296.

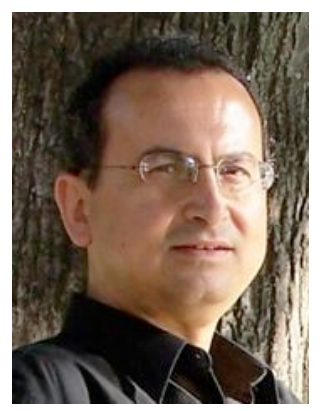

Massimo Rogante has been working in the field of neutron scattering for over 25 years. Degree in Mechanical Engineering at the Ancona University, Italy, he obtained his $\mathrm{PhD}$ in Nuclear Engineering at the Bologna University, Italy (1999); Director of the Rogante Engineering Office, operating in industrial applications of neutron techniques; Member of the International Scientific Advisory Committee of the Budapest Neutron Centre (BNC) since 2007; associated and consulting Editor and referee of various international scientific journals. Dr. Rogante is experienced in: investigations of materials and components from industrial and cultural Heritage sectors, by Neutron Techniques; Decommissioning of Nuclear Facilities and Nuclear Installations; materials processing; technology transfer. Co-founder of various companies; he is speaker at the Central-European Neutron Training School (CETS). He is author/co-author of 180 publications, member of the Academia Georgica Treiensis (Italy), the Accademia de' Catenati (Italy), the Nuclear Energy Institute (NEI), as well as several other international and domestic scientific bodies. Participation, as speaker, invited lecturer, Committee member and Co-organizer, to several International Conferences and Workshops. Further information can be found at the web site www.roganteengineering.com.

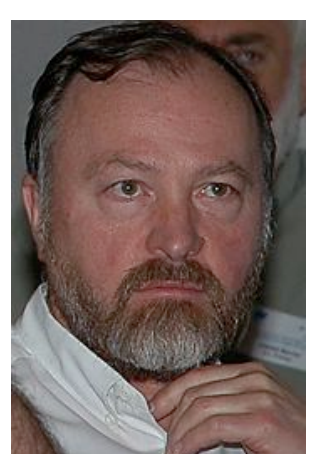

László Rosta has been working in the field of neutron scattering for over 40 years. He has been graduated from the Moscow State Lomonosov University (1973), obtained his $\mathrm{PhD}$ in Physics at the Budapest Eötvös Loránd University (1976); Research Fellow at the French Atomic Energy Commission (1980-87), visiting professor at the Vienna University (1992-93). $\mathrm{He}$ is leading the scientific activity at the Budapest Neutron Centre (BNC) since 1992. He has initiated and participated in the realisation of the research instrument suite at the $10 \mathrm{MW}$ Budapest Research Reactor, including a cold neutron source, neutron guide system and various spectrometers. He has gained experience in studying structure and dynamics of materials by various neutron and other techniques (X-ray, Raman, NMR etc.). In particular, small angle neutron scattering has been extensively used to reveal nano-scale features in materials like ferrofluids, biological membranes, bio-compatible ceramics or in industrial applications such as welds, turbines. Using neutrons as non-destructive tool for investigating objects of cultural Heritage is also in the scope of his recent interest. Dr. Rosta is experienced in technology transfer, co-founder of several spin-off companies; he has established the Central-European Neutron Training School (CETS). He is author/co-author of 270 publications, "Chevalier dans l'Ordre des Palmes Académique" (France), member of the Academia Georgica Treiensis (Italy) as well as numerous international and domestic scientific bodies. 